\title{
Matrix-Type Network DEA Model with Its Application Based on Input-Output Tables
}

\author{
Yun Cheng ${ }^{1}$ and Huan-Li Gao ${ }^{2}$ \\ ${ }^{1}$ School of Mechanical, Electrical \& Information Engineering, Shandong University at Weihai, Weihai 264209, China \\ ${ }^{2}$ School of Automation Science and Engineering, South China University of Technology, Guangzhou, China \\ Correspondence should be addressed to Yun Cheng; chy81@wh.sdu.edu.cn
}

Received 6 August 2014; Revised 25 November 2014; Accepted 1 December 2014

Academic Editor: Jinhui Zhang

Copyright (c) 2015 Y. Cheng and H.-L. Gao. This is an open access article distributed under the Creative Commons Attribution License, which permits unrestricted use, distribution, and reproduction in any medium, provided the original work is properly cited.

The matrix-type network data envelopment analysis (DEA) model is established for evaluating the relative performance of the matrix-type structure. The existence of solution and property of the new model is given. The equivalence of DEA efficiency and Pareto solutions of corresponding objective programming problem is proved. Using data in input-output tables, the new model is tested and the results show that the new model can be feasible in evaluating the relative performance of the matrix-type structure.

\section{Introduction}

In the process of enterprise, logistics, and supply chain management, we tend to analyze complex systems such as supply chain systems based on life cycle assessment (LCA), inputoutput systems, and others. The structure of the systems can be shown in Figure 1 and the efficiency scores of these systems are often required to be evaluated for the need of management decision making.

The generally accepted method for evaluating the relative performance of a set of comparable decision making units (DMUs) is data envelopment analysis (DEA). The first DEA model was introduced in 1978 [1] and several classic DEA models have been proposed over the past thirty years [2-5]. In recent years, DEA models were applied to evaluate the efficiency scores of complex systems and the concept of network DEA was put forward. The first network DEA model was introduced in 2000 [6], and then different models were put forward according to different system structure. The representative work includes models for series systems [5, 7-14], models for the parallel system [15-20], and model for complex system containing multiple subsystems introduced by Amatatsu and Ueda [21], Wang et al. [22], and Zhao et al. [23]. However, the models above cannot efficiently evaluate the efficiency score of the system shown in Figure 1.
In the following sections, a new model for evaluating the efficiency score of the system shown in Figure 1 is presented. As the model can be transformed into the linear programming problem, the existence of solution and property of the new model will be given. Computational experiments will be also presented to study the performances of the proposed model.

\section{Network DEA Model for Matrix-Type Organizations}

In the system shown in Figure 1, each subsystem has its own external inputs and external outputs, produces goods for other subsystems, and receives goods from other subsystems simultaneously. We refer to this type of system as a matrixtype system. Because of the complex internal structure, the relative performance of the system cannot be evaluated by the models mentioned above.

Consider the subsystem $s_{l}(l=1,2, \ldots, n)$ of $\mathbf{D M U}_{j}(j=$ $1,2, \ldots, m)$ shown in Figure 2 . Here, $x_{j}^{l}, y_{j}^{l}(l=1,2, \ldots, n ; j=$ $1,2, \ldots, m)$, respectively, represent the external inputs and outputs for the subsystem $s_{l}(l=1,2, \ldots, n)$ of $\operatorname{DMU}_{j}(j=$ $1,2, \ldots, m) ; z_{j}^{(k, l)}(k=1,2, \ldots, m, k \neq l)$ represent the internal inputs from other subsystems; and $z_{j}^{(l, k)}$ 


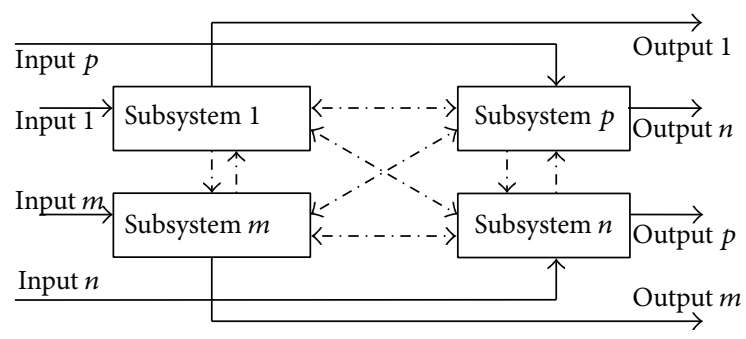

FIGURE 1: Matrix-type structure.

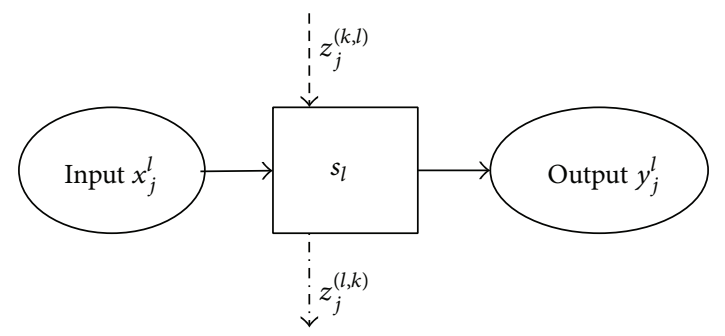

FIgURe 2: Subsystem $s_{l}$ of $\mathbf{D M U}_{j}$.

$(k=1,2, \ldots, m, k \neq l)$ represent the internal outputs to other subsystems.

Let $x_{0}^{l}=x_{j_{0}}^{l}, y_{0}^{l}=y_{j_{0}}^{l}, z_{0}^{(k, l)}=z_{j_{0}}^{(k, l)}$, and $z_{0}^{(l, k)}=z_{j_{0}}^{(l, k)}(l=$ $1,2, \ldots, n ; k=1,2, \ldots, n)$; the efficiency score of subsystem $s_{l}(l=1,2, \ldots, n)$ in $\mathbf{D M U}_{j_{0}}\left(j_{0} \in\{1,2, \ldots, n\}\right)$ can be gotten through the following model:

$$
\begin{array}{ll}
\max & \Psi_{l}=\frac{u_{l} y_{0}^{l}+\sum_{k=1, k \neq l}^{m} \xi_{l k} z_{0}^{(l, k)}}{v_{l} x_{0}^{l}+\sum_{k=1, k \neq l}^{m} \varsigma_{k l} z_{0}^{(k, l)}} \\
\text { s.t. } & \frac{u_{l} y_{j}^{l}+\sum_{k=1, k \neq l}^{m} \xi_{l k} z_{j}^{(l, k)}}{v_{l} x_{j}^{l}+\sum_{k=1, k \neq l}^{m} \varsigma_{k l} z_{j}^{(k, l)}} \leq 1, \quad j=1,2, \ldots, m, \\
& u_{l}, v_{l}, \xi_{l k}, \varsigma_{k l} \geq 0, \quad k=1,2, \ldots, m, k \neq l,
\end{array}
$$

where $v_{l}, \varsigma_{k l}$ represent the measure of the inputs and $u_{l}, \xi_{l k}$ represent the measure of the outputs. The optimal values of these parameters can be obtained by the optimization problem (1).

Considering the relationship among the inputs and outputs of subsystems in matrix-type structure, we can get the flow balance as follows:

$$
\sum_{k=1, k \neq l}^{m} \varsigma_{k l} z_{j}^{(k, l)}=\sum_{k=1, k \neq l}^{m} \xi_{k l} z_{j}^{(k, l)} .
$$

Then the model in (1) can be rewritten to the following form:

$$
\begin{array}{ll}
\max & \Psi^{l}=\frac{u_{l} y_{0}^{l}+\sum_{k=1, k \neq l}^{m} \xi_{l k} z_{0}^{(l, k)}}{v_{l} x_{0}^{l}+\sum_{k=1, k \neq l}^{m} \xi_{k l} z_{0}^{(k, l)}} \\
\text { s.t. } & \frac{u_{l} y_{j}^{l}+\sum_{k=1, k \neq l}^{m} \xi_{l k} z_{j}^{(l, k)}}{v_{l} x_{j}^{l}+\sum_{k=1, k \neq l}^{m} \xi_{k l} z_{j}^{(k, l)}} \leq 1, \quad j=1,2, \ldots, n, \\
& u_{l}, v_{l}, \xi_{l k} \geq 0, \quad k=1,2, \ldots, m, k \neq l .
\end{array}
$$

According to the properties of CCR model [1], we can get $\Psi^{l^{*}} \leq 1$ which is the optimal value of subsystem $s_{l}(l=$ $1,2, \ldots, n)$ and the following definition can be made.

Definition 1. If $\Psi^{l^{*}}=1$ and $\mu_{l}^{*}, \omega_{l}^{*}>0(k=1,2, \ldots, m, k \neq$ l) in model (3), the subsystem $s_{l}(l=1,2, \ldots, n)$ of $\mathbf{D M U}_{j_{0}}\left(j_{0} \in\{1,2, \ldots, n\}\right)$ is DEA efficient.

Furthermore, the efficiency score of $\mathbf{D M U}_{j_{0}}$ can be obtained through averaging the efficiency score of each subsystem $s_{l}(l=1,2, \ldots, n)$ with certain weight [17], which is the proportion of all inputs of subsystem $s_{l}(l=1,2, \ldots, n)$ which accounted for the entire inputs of the system. Then the efficiency score of $\mathbf{D M U}_{j_{0}}\left(j_{0} \in\{1,2, \ldots, n\}\right)$ can be evaluated by the following model:

$\max$

$$
\Phi=\sum_{l=1}^{m} \alpha_{l} \Psi^{l}
$$

$$
\begin{array}{ll}
\text { s.t. } & \frac{u_{l} y_{j}^{l}+\sum_{k=1, k \neq l}^{m} \xi_{l k} z_{j}^{(l, k)}}{v_{l} x_{j}^{l}+\sum_{k=1, k \neq l}^{m} \xi_{k l} z_{j}^{(k, l)}} \leq 1, \\
& j=1,2, \ldots, n ; \quad l=1,2, \ldots, m, \\
& u_{l}, v_{l}, \xi_{l k} \geq 0, \quad l=1,2, \ldots, m ; k=1,2, \ldots, m, k \neq l .
\end{array}
$$

Here, $\alpha_{l}=\left(v_{l} x_{0}^{l}+\sum_{k=1, k \neq l}^{m} \xi_{k l} z_{0}^{(k, l)}\right) / \sum_{l=1}^{m}\left(v_{l} x_{0}^{l}+\right.$ $\left.\sum_{k=1, k \neq l}^{m} \xi_{k l} z_{0}^{(k, l)}\right)$ represents the percentage of the subsystems' inputs in the total inputs.

Then we can get

$$
\begin{aligned}
\sum_{l=1}^{m} \alpha_{l} \Psi^{l}= & \sum_{l=1}^{m} \frac{v_{l} x_{0}^{l}+\sum_{k=1, k \neq l}^{m} \xi_{k l} z_{0}^{(k, l)}}{\sum_{l=1}^{m}\left(v_{l} x_{0}^{l}+\sum_{k=1, k \neq l}^{m} \xi_{k l} z_{0}^{(k, l)}\right)} \\
& * \frac{u_{l} y_{0}^{l}+\sum_{k=1, k \neq l}^{m} \xi_{l k} z_{0}^{(l, k)}}{v_{l} x_{0}^{l}+\sum_{k=1, k \neq l}^{m} \xi_{k l} z_{0}^{(k, l)}} \\
= & \frac{\sum_{l=1}^{m}\left(u_{l} y_{0}^{l}+\sum_{k=1, k \neq l}^{m} \xi_{l k} z_{0}^{(l, k)}\right)}{\sum_{l=1}^{m}\left(v_{l} x_{0}^{l}+\sum_{k=1, k \neq l}^{m} \xi_{k l} z_{0}^{(k, l)}\right)} ;
\end{aligned}
$$


model (4) can be rewritten as

$$
\begin{array}{ll}
\max & \Phi=\sum_{l=1}^{m} \alpha_{l} \Psi^{l}=\frac{\sum_{l=1}^{m}\left(u_{l} y_{0}^{l}+\sum_{k=1, k \neq l}^{m} \xi_{l k} z_{0}^{(l, k)}\right)}{\sum_{l=1}^{m}\left(v_{l} x_{0}^{l}+\sum_{k=1, k \neq l}^{m} \xi_{k l} z_{0}^{(k, l)}\right)} \\
\text { s.t. } & \frac{u_{l} y_{j}^{l}+\sum_{k=1, k \neq l}^{m} \xi_{l k} z_{j}^{(l, k)}}{v_{l} x_{j}^{l}+\sum_{k=1, k \neq l}^{m} \xi_{k l} z_{j}^{(k, l)} \leq 1,} \\
& j=1,2, \ldots, n ; \quad l=1,2, \ldots, m, \\
& u_{l}, v_{l}, \xi_{l k} \geq 0, \quad l=1,2, \ldots, m ; k=1,2, \ldots, m, k \neq l .
\end{array}
$$

To reduce model (6) to an ordinary linear programming problem, we rescale all data by means of the following formula:

$$
\begin{aligned}
& t=\frac{1}{\sum_{l=1}^{m}\left(v_{l} x_{0}^{l}+\sum_{k=1, k \neq l}^{m} \xi_{k l} z_{0}^{(k, l)}\right)}, \\
& \mu_{l}=t u_{l}, \quad \omega_{l}=t v_{l}, \quad \eta_{l k}=t \xi_{l k}, \\
& (l, k=1,2, \ldots, n) .
\end{aligned}
$$

Using these rescaled data in model (6), we obtain

$$
\begin{array}{ll}
\max & \Phi=\sum_{l=1}^{m}\left(\mu_{l} y_{0}^{l}+\sum_{k=1, k \neq l}^{m} \eta_{l k} z_{0}^{(l, k)}\right) \\
\text { s.t. } & \sum_{l=1}^{m}\left(\omega_{l} x_{0}^{l}+\sum_{k=1, k \neq l}^{m} \eta_{k l} z_{0}^{(k, l)}\right)=1, \\
& \mu_{l} y_{j}^{l}+\sum_{k=1, k \neq l}^{m} \eta_{l k} z_{j}^{(l, k)} \leq \omega_{l} x_{j}^{l}+\sum_{k=1, k \neq l}^{m} \eta_{k l} z_{j}^{(k, l)}, \\
& j=1,2, \ldots, n ; \quad l=1,2, \ldots, m, \\
& \mu_{l}, \omega_{l}, \eta_{l k} \geq 0, \quad l=1,2, \ldots, m ; k=1,2, \ldots, m, k \neq l .
\end{array}
$$

Obviously, the optimal objective values of both model (6) and model (8) are equal.

The dual problem of model (8) can be expressed as

$$
\begin{array}{ll}
\min & \theta \\
\text { s.t. } & \sum_{j=1}^{n} x_{j}^{l} \lambda_{j}^{l} \leq \theta x_{0}^{l}, \quad l=1,2, \ldots, m, \\
& \sum_{j=1}^{n} y_{j}^{l} \lambda_{j}^{l} \geq y_{0}^{l}, \quad l=1,2, \ldots, m, \\
& \sum_{j=1}^{n} z_{j}^{(k, l)} \lambda_{j}^{l}-\sum_{j=1}^{n} z_{j}^{(k, l)} \lambda_{j}^{k} \leq \theta z_{0}^{(k, l)}-z_{0}^{(k, l),} \\
& l=1,2, \ldots, m, \quad k=1,2, \ldots, m, \quad k \neq l, \\
& \lambda_{j}^{l} \geq 0, \quad l=1,2, \ldots, m, \quad j=1,2, \ldots, n .
\end{array}
$$

\section{Property of New Model}

Theorem 2. The optimal solution of model (8) exists and the optimal objective values $\Phi^{*} \leq 1$.

Proof. See the Appendix.

Definition 3. The $\mathbf{D M U}_{j_{0}}\left(j_{0} \in\{1,2, \ldots, n\}\right)$ is DEA efficient if the optimal objective value of model (8) is 1 and $\mu_{l}^{*}, \omega_{l}^{*}>0$, $l=1,2, \ldots, m$.

According to the duality theorem and elastic theorem of linear programming, we can get the following.

Definition 4. The $\mathbf{D M U}_{j_{0}}\left(j_{0} \in\{1,2, \ldots, n\}\right)$ is DEA efficient if the optimal solutions of model (9) which can be represented as $\theta^{*}, \lambda_{j}^{l *}(j=1,2, \ldots, n ; l=1,2, \ldots, m)$ meet

$$
\begin{aligned}
\theta^{*}=1, \quad \sum_{j=1}^{n} x_{j}^{l} \lambda_{j}^{l *}=\theta^{*} x_{0}^{l}, \quad & \sum_{j=1}^{n} y_{j}^{l} \lambda_{j}^{l *}=y_{0}^{l}, \\
& l=1,2, \ldots, m .
\end{aligned}
$$

Theorem 5. The $\mathbf{D M U}_{j_{0}}\left(j_{0} \in\{1,2, \ldots, n\}\right)$ is DEA efficient if and only if all subsystems of the $\mathbf{D M U}_{j_{0}}$ are DEA efficient.

Proof. See the Appendix.

\section{Equivalence of DEA Efficiency and Pareto Solution}

Consider the following multiple objective programming problems:

$$
\begin{aligned}
\min F(\mathbf{X}, \mathbf{Y}) & =(\mathbf{X},-\mathbf{Y}) \\
& =\left(x^{1}, x^{2}, \ldots, x^{m} ;-y^{1},-y^{2}, \ldots,-y^{m}\right)^{T}
\end{aligned}
$$

s.t. $(\mathbf{X}, \mathbf{Y}) \in T$.

Here,

$$
\begin{gathered}
T=\left\{(\mathbf{X}, \mathbf{Y}) \mid \sum_{j=1}^{n} x_{j}^{l} \lambda_{j}^{l} \leq x^{l}, \sum_{j=1}^{n} y_{j}^{l} \lambda_{j}^{l} \geq y^{l},\right. \\
\sum_{j=1}^{n} z_{j}^{(k, l)} \lambda_{j}^{l} \leq \sum_{j=1}^{n} z_{j}^{(k, l)} \lambda_{j}^{k}, \\
l=1,2, \ldots, m ; k=1,2, \ldots, m, k \neq l\}
\end{gathered}
$$

is the production possibility set of the matrix-type DEA model.

Definition 6. Let $\left(\mathbf{X}^{*}, \mathbf{Y}^{*}\right) \in T$ and if there does not exist $(\mathbf{X}, \mathbf{Y}) \in T$ which makes $F(\mathbf{X}, \mathbf{Y}) \leq F\left(\mathbf{X}^{*}, \mathbf{Y}^{*}\right),\left(\mathbf{X}^{*}, \mathbf{Y}^{*}\right)$ is defined as the Pareto solution of model (11). 


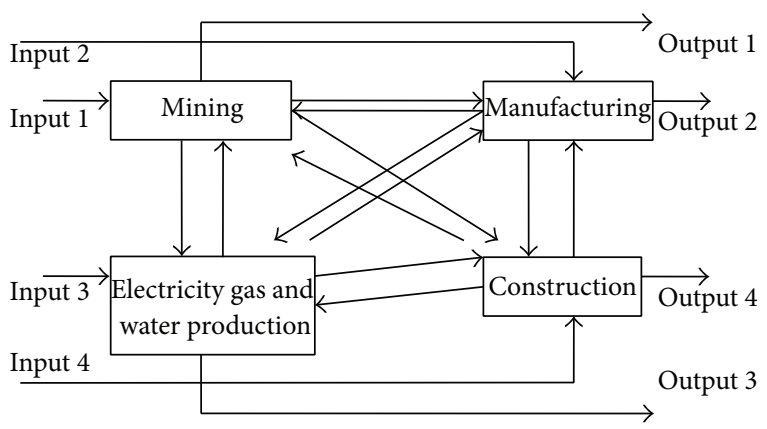

FIgURE 3: The construction of the second industry.

Lemma 7. If $\left(\mathbf{X}_{\mathbf{0}}, \mathbf{Y}_{\mathbf{0}}\right)$ is the optimal solution of $\min _{(\mathbf{X}, \mathbf{Y}) \in T}\left(\boldsymbol{\omega}^{\mathbf{0 T}} \mathbf{X}-\boldsymbol{\mu}^{\mathbf{0 T}} \mathbf{Y}\right)$ and $\left(\boldsymbol{\omega}^{0 \mathrm{~T}}, \boldsymbol{\mu}^{\mathbf{0 T}}\right)>\mathbf{0},\left(\mathbf{X}_{\mathbf{0}}, \mathbf{Y}_{\mathbf{0}}\right)$ is the Pareto solution of model (11).

Proof. See the Appendix.

Theorem 8. The $\mathbf{D M U}_{j_{0}}\left(j_{0} \in\{1,2, \ldots, n\}\right)$ is DEA efficient if and only if $\left(\mathbf{X}_{\mathbf{0}}, \mathbf{Y}_{\mathbf{0}}\right)$ is the Pareto solution of model (11).

Proof. See the Appendix.

\section{Analysis of Efficiencies of the Second Industry of 27 Provinces in China Based on Input-Output Tables}

Input-output tables are fundamental statistical data in economic, social, and environmental issues. Chiang et al. applied black box DEA programs to input-output tables [24]. Jiang et al. analyzed the national economy efficiency through inputoutput tables with the DEA method [25] and Amatatsu and Ueda applied the new SBM model to input-output tables of 47 prefectures in Japan and assessed the industrial efficiencies of them [21].

In this part, we apply the matrix model to input-output tables. We consider the efficiency scores of the second industry of 27 provinces in China in 2007. Referring to the statistics specification of the National Bureau of China, there are four sectors in the second industry including mining, manufacturing, electricity gas and water production, and construction. Taking Shanxi for example, the data of four sectors input-output table can be listed in Table 1.

The construction of the second industry can be shown in Figure 3. Conventional DEA models construction as a "black box" as shown in Figure 4.

From Figure 3, we can see that the efficiency scores of the second industry of these provinces can be calculated by the matrix-type model. Using model (8), the efficiency scores of the 27 provinces in China can be shown in Table 2. Then, each sector's scores can be obtain by the formula

$$
\Phi_{l}=\mu_{l}^{*} y_{0}^{l}+\sum_{k=1, k \neq l}^{m} \eta_{l k}^{*} z_{0}^{(l, k)}
$$

and the scores are also shown in Table 2.

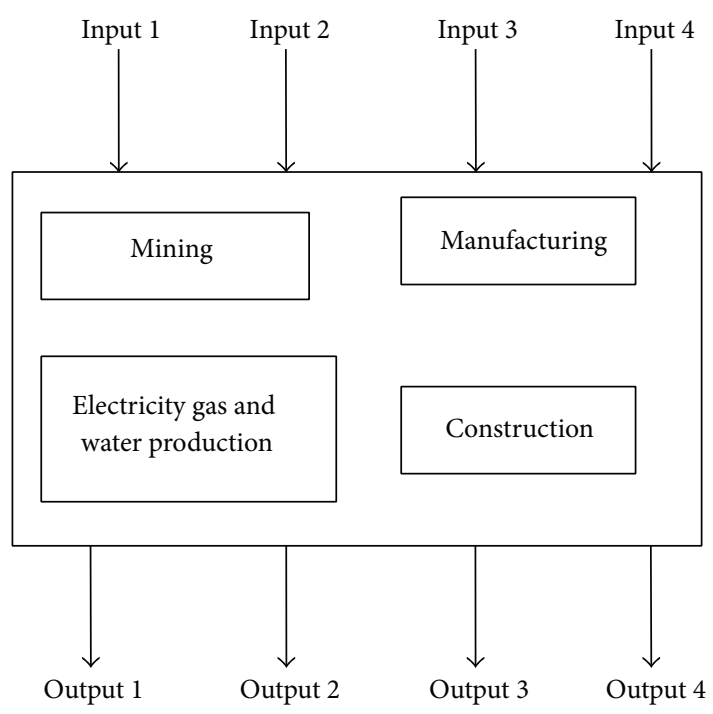

Figure 4: The traditional "black model."

It can be seen that the efficiency scores obtained from the matrix-type model are not accurate for ignoring the internal inputs and outputs among the subsystems. There are five DMUs' efficiency scores which are equal to 1 in black model and only the efficiency scores of Henan province are equal to 1 in our new model.

The new model can not only calculate the more accurate efficiency scores of the DMUs but also give the efficiency score of each subsystem which provides detailed information for the decision makers. As Hebei, the efficiency score got by black model is 0.386 . Analyzing the efficiency score got by matrix model, not all the sectors are inefficient. The mining and the electricity gas and water production are both DEA efficient, and the low efficiency score is because of the manufacture and constructor.

\section{Conclusions}

This paper has established a matrix-type DEA model for matrix-type organization and proved the existence of solution. Also, the property of the new model and the equivalence of DEA efficiency and Pareto solutions of corresponding objective programming problem are given. Then the new model has been applied to the input-output tables and got the meaningful conclusions.

A point that should be stressed is that the new model considers the internal linking activities, and the influence of the interaction of the subsystems on the whole efficiency score is represented. Based on model (8), the relative performance of each subsystem can be evaluated. In contrast to the black model, the new model gives more accurate result.

Finally, in addition to input-output tables, cycle industry is also typical matrix-type organization, such as cycle automobile industry which includes production, marketing, repair and recovery, the four sectors are influenced each other. We will give special discussion on the efficiency of this kind of industry in the further study. 
TABLE 1: Example of four sectors of Shanxi's I-O table (unit: billion).

\begin{tabular}{|c|c|c|c|c|c|}
\hline & Mining & Manufacturing & Electricity gas and water production & Construction & Output \\
\hline Mining & 3.68 & 65.26 & 11.41 & 20.2 & 96.72 \\
\hline Manufacturing & 20.9 & 209.6 & 3.94 & 82.2 & 572.3 \\
\hline Electricity gas and water production & 5.49 & 20.4 & 8.72 & 1.85 & 25.3 \\
\hline Construction & 0.18 & 0.24 & 0.023 & 0 & 293.79 \\
\hline Input & 132.4 & 55.7 & 24.3 & 57.3 & \\
\hline
\end{tabular}

TABLE 2: Efficiency scores obtained from the "black model" and new model.

\begin{tabular}{|c|c|c|c|c|c|}
\hline \multirow[t]{2}{*}{$\mathbf{D M U}_{j}$} & \multirow[t]{2}{*}{ Black box model } & \multicolumn{4}{|c|}{$\begin{array}{c}\text { Matrix model } \\
\text { Subsystem }\end{array}$} \\
\hline & & Primary & Secondary & Tertiary & Quaternary \\
\hline Anhui & 0.26 & 0.584 & 0.683 & 0.518 & 0.629 \\
\hline Beijing & 0.156 & 0.586 & 0.604 & 1 & 0.025 \\
\hline Chongqing & 0.507 & 0.446 & 0.634 & 0.789 & 0.498 \\
\hline Fujian & 0.328 & 0.456 & 0.402 & 0.266 & 0.674 \\
\hline Gansu & 0.143 & 0.543 & 0.558 & 0.171 & 0.833 \\
\hline Guangdong & 0.576 & 0.655 & 1 & 0.301 & 0.249 \\
\hline Guangxi & 0.161 & 0.535 & 0.485 & 0.368 & 0.762 \\
\hline Guizhou & 0.141 & 0.636 & 0.482 & 0.503 & 0.819 \\
\hline Hainan & 1 & 0.603 & 0.211 & 1 & 0.887 \\
\hline Heilongjiang & 1 & 0.706 & 0.88 & 0.904 & 0.325 \\
\hline Henan & 1 & 1 & 1 & 1 & 1 \\
\hline Hubei & 0.313 & 0.596 & 1 & 0.478 & 1 \\
\hline Hunan & 0.089 & 0.482 & 0.66 & 0.188 & 0.562 \\
\hline Inner Mongolia & 0.268 & 0.439 & 0.423 & 0.268 & 0.496 \\
\hline Jiangsu & 0.274 & 0.732 & 0.975 & 0.557 & 1 \\
\hline Jiangxi & 0.32 & 0.5 & 0.669 & 0.915 & 0.63 \\
\hline Jilin & 0.222 & 0.435 & 0.514 & 0.552 & 1 \\
\hline Liaoning & 0.19 & 0.46 & 0.405 & 0.387 & 0.509 \\
\hline Ningxia & 1 & 0.822 & 0.871 & 0.012 & 0.067 \\
\hline Shaanxi & 0.512 & 0.566 & 0.976 & 0.64 & 0.478 \\
\hline Shandong & 0.174 & 0.61 & 0.186 & 0.546 & 1 \\
\hline Shanxi & 1 & 0.716 & 0.594 & 0.934 & 0.456 \\
\hline Tianjin & 0.399 & 0.476 & 0.266 & 0.426 & 0.585 \\
\hline Xinjiang & 0.612 & 0.447 & 0.282 & 0.578 & 0.372 \\
\hline Yunnan & 0.334 & 0.511 & 0.619 & 0.573 & 0.579 \\
\hline Zhejiang & 0.241 & 0.528 & 0.93 & 0.174 & 0.755 \\
\hline
\end{tabular}

\section{Appendix}

Proof of Theorem 2. Let $\bar{\theta}=1, \bar{\lambda}_{j}^{l}=\left\{0, j \neq j_{0} ; 1, j=j_{0}\right\}$, $l=1,2, \ldots, m$; it is easy to see that $\bar{\theta}_{l}, \bar{\lambda}_{j}^{l}, l=1,2, \ldots, m, j=$ $1,2, \ldots, n$, is the feasible solutions of model (9). Referring to the linear programming optimal solution existence theorem, we can know that the optimal solutions of model (9) and model (8) exist. Denote by

$$
\begin{aligned}
& \omega_{1}^{*}, \ldots, \omega_{m}^{*}, \mu_{1}^{*}, \ldots, \mu_{m}^{*}, \eta_{21}^{*}, \eta_{31}^{*}, \ldots, \eta_{m 1}^{*}, \\
& \eta_{12}^{*}, \eta_{32}^{*}, \ldots, \eta_{m 2}^{*}, \ldots, \eta_{1 m}^{*}, \eta_{2 m}^{*}, \ldots, \eta_{m-1, m}^{*}
\end{aligned}
$$

the optimal solutions of model (8); we then obtain

$$
\begin{gathered}
-\omega_{l}^{*} x_{0}^{l}+\mu_{l}^{*} y_{0}^{l}-\sum_{k=1, k \neq l}^{m} \eta_{k l}^{*} z_{0}^{(k, l)}+\sum_{k=1, k \neq l}^{m} \eta_{l k}^{*} z_{0}^{(l, k)} \leq 0, \\
l=1,2, \ldots, m, \\
\sum_{l=1}^{m}\left(\omega_{l}^{*} x_{0}^{l}+\sum_{k=1, k \neq l}^{m} \eta_{k l}^{*} z_{0}^{(k, l)}\right)=1 .
\end{gathered}
$$


From (A.2), we can get

$$
\sum_{l=1}^{m}\left(-\omega_{l}^{*} x_{0}^{l}+\mu_{l}^{*} y_{0}^{l}-\sum_{k=1, k \neq l}^{m} \eta_{k l}^{*} z_{0}^{(k, l)}+\sum_{k=1, k \neq l}^{m} \eta_{l k}^{*} z_{0}^{(l, k)}\right) \leq 0 .
$$

Considering (A.3) and $\sum_{l=1}^{m} \sum_{k=1, k \neq l}^{m} \eta_{k l}^{*} z_{0}^{(k, l)}=$ $\sum_{l=1}^{m} \sum_{k=1, k \neq l}^{m} \eta_{l k}^{*} z_{0}^{(l, k)}$, (A.4) can be rewritten as

$$
1-\sum_{l=1}^{m} \sum_{k=1, k \neq l}^{m} \eta_{k l}^{*} z_{0}^{(k, l)}+\sum_{l=1}^{m} \mu_{l}^{*} y_{0}^{l} \leq 0
$$

Then $\Phi^{*}=\sum_{l=1}^{m}\left(\mu_{l}^{*} y_{0}^{l}+\sum_{k=1, k \neq l}^{m} \eta_{l k}^{*} z_{0}^{(l, k)}\right) \leq 1$; the theorem is confirmed.

\section{Proof of Theorem 5.}

Sufficiency. If the subsystems of the $\mathbf{D M U}_{j_{0}}$ are all DEA efficient, there exist the optimal solutions $u_{l}^{*}>0, v_{l}^{*}>$ $0, \xi_{k l}^{*} \geq 0(k=1,2, \ldots, m, k \neq l)$ which make $\Psi^{l *}=$ $1(l=1,2, \ldots, m)$ for model (3). Considering the constraint conditions in model (3) and model (4), we can know that $u_{l}^{*}>$ $0, v_{l}^{*}>0, \xi_{k l}^{*} \geq 0(k=1,2, \ldots, m, k \neq l)$ are also the feasible solutions of model (4). According to those feasible solutions, the value of model (4) is $\Phi^{*}=\sum_{l=1}^{m} \alpha_{l} \Psi^{l *}=\sum_{l=1}^{m} \alpha_{l}=1$.

Combined with Theorem $2, \Phi^{*}=1$ is the optimal solutions of model (4) and is also the optimal solutions of model (8); then the $\mathbf{D M U}_{j_{0}}\left(j_{0} \in\{1,2, \ldots, n\}\right)$ is DEA efficient.

Necessity. If the $\mathbf{D M U}_{j_{0}}\left(j_{0} \in\{1,2, \ldots, n\}\right)$ is DEA efficient, the optimal solutions $u_{l}^{*}>0, v_{l}^{*}>0, \xi_{k l}^{*} \geq 0, l=$ $1,2, \ldots, m, k=1,2, \ldots, m, k \neq l$, of model (4) exist which make $\Phi^{*}=\sum_{l=1}^{m} \alpha_{l} \widehat{\Psi}^{l *}=1$.

According to $\sum_{l=1}^{m} \alpha_{l}=1$ and $\widehat{\Psi}^{l *} \leq 1, l=1,2, \ldots, m$, we can get $\widehat{\Psi}^{l *}=1, l=1,2, \ldots, m$. Considering the constraint conditions in model (3) and model (4), we know that $u_{l}^{*}>$ $0, v_{l}^{*}>0, \xi_{k l}^{*} \geq 0, k=1,2, \ldots, m, k \neq l$, are the feasible solutions of model (3) when $l=1,2, \ldots, m$, respectively. Then there are $\Psi^{l *}=\widehat{\Psi}^{l *}=1, l=1,2, \ldots, m$, and the subsystems of the $\mathbf{D M U}_{j_{0}}\left(j_{0} \in\{1,2, \ldots, n\}\right)$ are all DEA efficient.

Proof of Lemma 7. If $\left(\mathbf{X}_{\mathbf{0}}, \mathbf{Y}_{\mathbf{0}}\right)$ is not the Pareto solution of model (11), then there exists $(\widehat{\mathbf{X}}, \widehat{\mathbf{Y}}) \in T$ which makes $\left(\begin{array}{c}\widehat{\mathbf{X}} \\ -\widehat{\mathbf{Y}}\end{array}\right) \leq$ $\left(\begin{array}{c}\mathbf{X}_{0} \\ -\mathbf{Y}_{0}\end{array}\right)$. Let $\left(\omega^{0 \mathrm{~T}}, \boldsymbol{\mu}^{0 \mathrm{~T}}\right)>\mathbf{0}$; we have

$$
\left(\omega^{0 \mathrm{~T}}, \boldsymbol{\mu}^{0 \mathrm{~T}}\right)\left[\left(\begin{array}{c}
\widehat{\mathrm{X}} \\
-\widehat{\mathrm{Y}}
\end{array}\right)-\left(\begin{array}{c}
\mathbf{X}_{0} \\
-\mathbf{Y}_{0}
\end{array}\right)\right] \leq 0
$$

Then $\boldsymbol{\omega}^{0 \mathrm{~T}} \widehat{\mathbf{X}}-\boldsymbol{\mu}^{0 \mathrm{~T}} \widehat{\mathbf{Y}} \leq \boldsymbol{\omega}^{0 \mathrm{~T}} \mathbf{X}_{\mathbf{0}}-\boldsymbol{\mu}^{0 \mathrm{~T}} \mathbf{Y}_{0}$, which is contrary to the hypothesis that $\left(\mathbf{X}_{\mathbf{0}}, \mathbf{Y}_{\mathbf{0}}\right)$ is the optimal solution of $\min _{(X, Y) \in T}\left(\boldsymbol{\omega}^{\mathbf{0 T}} \mathbf{X}-\boldsymbol{\mu}^{\mathbf{0 T}} \mathbf{Y}\right)$.

\section{Proof of Theorem 8.}

Sufficiency. If the $\mathbf{D M U}_{j_{0}}$ is DEA efficient, the optimal solutions of model (8) exist which are denoted by $\omega_{l}^{*}>0$, $\mu_{l}^{*}>0, \eta_{k l}^{*} \geq 0(k=1,2, \ldots, m, k \neq l)$ and the corresponding optimal value is $\Phi^{*}=1$. Then,

$$
\begin{gathered}
\sum_{l=1}^{m}\left(\mu_{l}^{*} y_{0}^{l}+\sum_{k=1, k \neq l}^{m} \eta_{l k}^{*} z_{0}^{(l, k)}\right)=1, \\
\sum_{l=1}^{m}\left(\omega_{l}^{*} x_{0}^{l}+\sum_{k=1, k \neq l}^{m} \eta_{k l}^{*} z_{0}^{(k, l)}\right)=1, \\
-\omega_{l}^{*} x_{j}^{l}+\mu_{l}^{*} y_{j}^{l}-\sum_{k=1, k \neq l}^{m} \eta_{k l}^{*} z_{j}^{(k, l)}+\sum_{k=1, k \neq l}^{m} \eta_{l k}^{*} z_{j}^{(l, k)} \leq 0, \\
l=1,2, \ldots, m ; \quad j=1,2, \ldots, n .
\end{gathered}
$$

Let $\beta_{j}^{l} \geq 0, j=1,2, \ldots, n, l=1,2, \ldots, m$, and

$$
\begin{aligned}
& \sum_{j=1}^{n} z_{j}^{(k, l)} \beta_{j}^{l} \leq \sum_{j=1}^{n} z_{j}^{(k, l)} \beta_{j}^{k}, \\
& l=1,2, \ldots, m ; \quad k=1,2, \ldots, m, \quad k \neq l .
\end{aligned}
$$

We can rewrite (A.9) as

$$
\begin{gathered}
\sum_{j=1}^{n} \sum_{l=1}^{m}\left(-\omega_{l}^{*} x_{j}^{l} \beta_{j}^{l}+\mu_{l}^{*} y_{j}^{l} \beta_{j}^{l}-\sum_{k=1, k \neq l}^{m} \eta_{k l}^{*} z_{j}^{(k, l)} \beta_{j}^{l}\right. \\
\left.+\sum_{k=1, k \neq l}^{m} \eta_{l k^{*}}^{*} z_{j}^{(l, k)} \beta_{j}^{l}\right) \leq 0 .
\end{gathered}
$$

Arranging (A.11) and considering

$$
\begin{aligned}
& \sum_{j=1}^{n} \sum_{l=1}^{m}\left(-\sum_{k=1, k \neq l}^{m} \eta_{k l}^{*} z_{j}^{(k, l)} \beta_{j}^{l}+\sum_{k=1, k \neq l}^{m} \eta_{l k}^{*} z_{j}^{(l, k)} \beta_{j}^{l}\right) \\
& =\sum_{j=1}^{n}\left(-\sum_{l=1}^{m} \sum_{k=1, k \neq l}^{m} \eta_{k l}^{*} z_{j}^{(k, l)} \beta_{j}^{l}+\sum_{l=1}^{m} \sum_{k=1, k \neq l}^{m} \eta_{l k}^{*} z_{j}^{(l, k)} \beta_{j}^{l}\right) \\
& =\sum_{j=1}^{n}\left(\sum_{l=1}^{m} \sum_{k=1, k \neq l}^{m} \eta_{k l}^{*} z_{j}^{(k, l)}\left(-\beta_{j}^{l}+\beta_{j}^{k}\right)\right) \geq 0,
\end{aligned}
$$

we can get $\sum_{j=1}^{n} \sum_{l=1}^{m}\left(-\omega_{l}^{*} x_{j}^{l} \beta_{j}^{l}+\mu_{l}^{*} y_{j}^{l} \beta_{j}^{l}\right) \leq 0$.

Applying (A.7) and equation $\sum_{l=1}^{m} \sum_{k=1, k \neq l}^{m} \eta_{l k}^{*} z_{0}^{(l, k)}=$ $\sum_{l=1}^{m} \sum_{k=1, k \neq l}^{m} \eta_{k l}^{*} z_{0}^{(k, l)}$ to (A.4), the equality $-\sum_{l=1}^{m} \omega_{l}^{*} x_{0}^{l}+$ $\sum_{l=1}^{m} \mu_{l}^{*} y_{0}^{l}=0$ is gotten.

That is,

$$
\sum_{j=1}^{n} \sum_{l=1}^{m}\left(\omega_{l}^{*} x_{j}^{l} \beta_{j}^{l}-\mu_{l}^{*} y_{j}^{l} \beta_{j}^{l}\right) \geq \sum_{l=1}^{m} \omega_{l}^{*} x_{0}^{l}-\sum_{l=1}^{m} \mu_{l}^{*} y_{0}^{l} .
$$

So, $\forall(\mathbf{X}, \mathbf{Y}) \in T$ we can get

$$
\begin{aligned}
\boldsymbol{\omega}^{* T} X-\boldsymbol{\mu}^{* T} Y & \geq \boldsymbol{\omega}^{* T} \sum_{j=1}^{n} x_{j}^{l} \lambda_{j}^{l}-\boldsymbol{\mu}^{* T} \sum_{j=1}^{n} y_{j}^{l} \lambda_{j}^{l} \\
& \geq \boldsymbol{\omega}^{* T} X_{0}-\boldsymbol{\mu}^{* T} Y_{0}
\end{aligned}
$$


where $\boldsymbol{\omega}^{*}=\left(\omega_{1}^{*}, \omega_{2}^{*}, \ldots, \omega_{m}^{*}\right)^{T}>0 ; \boldsymbol{\mu}^{*}=\left(\mu_{1}^{*}, \mu_{2}^{*}, \ldots, \mu_{m}^{*}\right)^{T}>$ 0 . Then $\left(\mathbf{X}_{\mathbf{0}}, \mathbf{Y}_{\mathbf{0}}\right)$ is the optimal solution of

$$
\begin{array}{ll}
\min & \boldsymbol{\omega}^{* \mathrm{~T}} \mathbf{X}-\boldsymbol{\mu}^{* \mathrm{~T}} \mathbf{Y} \\
\text { s.t. } & (\mathbf{X}, \mathbf{Y}) \in T .
\end{array}
$$

According to Lemma $7,\left(\mathbf{X}_{\mathbf{0}}, \mathbf{Y}_{\mathbf{0}}\right)$ is also the Pareto solution of model (11).

Necessity. We suppose $\left(\mathbf{X}_{\mathbf{0}}, \mathbf{Y}_{\mathbf{0}}\right)$ is the Pareto solution of model (11) and the $\mathbf{D M U} \mathbf{j}_{0}$ is not DEA efficient. The following situations can be gotten as follows.

(a) The optimal solution of model (9) is less than 1; that is, $\theta^{*}<1$.

(b) The optimal solution of model (9) is 1 and there exists at least 1 serial number $i$ which belongs to the array $\{1,2, \ldots, m\}$ and $\sum_{j=1}^{n} x_{j}^{i} \lambda_{j}^{i *}<\theta^{*} x_{0}^{i}$.

(c) The optimal solution of model (9) is 1 and there exists at least 1 serial number $i$ which belongs to the array $\{1,2, \ldots, m\}$ and $\sum_{j=1}^{n} y_{j}^{i} \lambda_{j}^{i *}>y_{0}^{i}$.

Now, we make the proof, respectively, according to the situations above.

(a) Let $\widehat{x}_{0}^{l}=\theta^{*} x_{0}^{l}<x_{0}^{l}, \widehat{\mathbf{X}}_{0}=\left\{\widehat{x}_{0}^{l}, \ldots, \widehat{x}_{0}^{l}, \ldots \widehat{x}_{0}^{n}\right\}$; then $\widehat{\mathbf{X}}_{\mathbf{0}}<\mathbf{X}_{\mathbf{0}}$. Considering the constraint conditions in model (9), we can get

$$
\begin{gathered}
\sum_{j=1}^{n} x_{j}^{l} \lambda_{j}^{* l} \leq \theta^{*} x_{0}^{l}=\widehat{x}_{0}^{l}, \quad l=1,2, \ldots m, \\
\sum_{j=1}^{n} y_{j}^{l} \lambda_{j}^{l *} \geq y_{0}^{l}, \quad l=1,2, \ldots m, \\
\sum_{j=1}^{n} z_{j}^{(k, l)} \lambda_{j}^{l *}-\sum_{j=1}^{n} z_{j}^{(k, l)} \lambda_{j}^{k *} \leq \theta^{*} z_{0}^{(k, l)}-z_{0}^{(k, l)} \leq 0, \\
l=1,2, \ldots, m, \quad k=1,2, \ldots, m, \quad k \neq l .
\end{gathered}
$$

Then $\left(\widehat{\mathbf{X}}_{\mathbf{0}}, \mathbf{Y}_{\mathbf{0}}\right)$ is the feasible solution of model (11) and $\left(\begin{array}{c}\widehat{\mathbf{X}}_{0} \\ -\mathbf{Y}_{0}\end{array}\right) \leq\left(\begin{array}{c}\mathbf{X}_{0} \\ -\mathbf{Y}_{0}\end{array}\right)$. The conclusion is contrary to the hypothesis before.

(b) There exists $s>0$ and $\sum_{j=1}^{n} x_{j}^{i} \lambda_{j}^{i *}+s=x_{0}^{i}$. Let $\widehat{x}_{0}^{i}=x_{0}^{i}-$ $s<x_{0}^{l}$ and $\widehat{\mathbf{X}}_{\mathbf{0}}=\left\{x_{0}^{1}, \ldots, \widehat{x}_{0}^{i}, \ldots x_{0}^{n}\right\}$; then we have $\widehat{\mathbf{X}}_{\mathbf{0}} \leq \mathbf{X}_{\mathbf{0}}$. Considering the constraint conditions in model (9), we can get

$$
\begin{gathered}
\sum_{j=1}^{n} x_{j}^{l} \lambda_{j}^{* l} \leq \theta^{*} x_{0}^{l}=x_{0}^{l}, \quad l=1,2, \ldots, m, \quad l \neq i, \\
\sum_{j=1}^{n} x_{j}^{i} \lambda_{j}^{* i}=\hat{x}_{0}^{i}, \\
\sum_{j=1}^{n} y_{j}^{l} \lambda_{j}^{l *} \geq y_{0}^{l}, \quad l=1,2, \ldots, m,
\end{gathered}
$$

$$
\begin{array}{r}
\sum_{j=1}^{n} z_{j}^{(k, l)} \lambda_{j}^{l *}-\sum_{j=1}^{n} z_{j}^{(k, l)} \lambda_{j}^{k *} \leq \theta^{*} z_{0}^{(k, l)}-z_{0}^{(k, l)} \leq 0, \\
\quad l=1,2, \ldots, m, \quad k=1,2, \ldots, m, \quad k \neq l .
\end{array}
$$

Then $\left(\widehat{\mathbf{X}}_{\mathbf{0}}, \mathbf{Y}_{\mathbf{0}}\right)$ is the feasible solution of model (9) and $\left(\begin{array}{c}\widehat{\mathbf{X}}_{0} \\ -\mathbf{Y}_{0}\end{array}\right) \leq\left(\begin{array}{c}\mathbf{X}_{0} \\ -\mathbf{Y}_{0}\end{array}\right)$. The conclusion is contrary to the hypothesis before.

(c) There exists $s>0$ and $\sum_{j=1}^{n} y_{j}^{i} \lambda_{j}^{i *}-s=y_{0}^{i}$. Let $\widehat{y}_{0}^{i}=y_{0}^{i}+$ $s>y_{0}^{i}$ and $\widehat{\mathbf{Y}}_{\mathbf{0}}=\left\{y_{0}^{1}, \ldots, \widehat{y}_{0}^{i}, \ldots, y_{0}^{n}\right\}$; then we have $\widehat{\mathbf{Y}}_{\mathbf{0}} \geq \mathbf{Y}_{\mathbf{0}}$. Considering the constraint conditions in model (9), we can get

$$
\begin{gathered}
\sum_{j=1}^{n} x_{j}^{l} \lambda_{j}^{* l} \leq \theta^{*} x_{0}^{l}=x_{0}^{l}, \quad l=1,2, \ldots, m, \\
\sum_{j=1}^{n} y_{j}^{l} \lambda_{j}^{l *} \geq y_{0}^{l}, \quad l=1,2, \ldots, m, \quad l \neq i, \\
\sum_{j=1}^{n} y_{j}^{i} \lambda_{j}^{i *}=\hat{y}_{0}^{i}, \quad l=1,2, \ldots, m, \\
\sum_{j=1}^{n} z_{j}^{(k, l)} \lambda_{j}^{l *}-\sum_{j=1}^{n} z_{j}^{(k, l)} \lambda_{j}^{k *} \leq \theta^{*} z_{0}^{(k, l)}-z_{0}^{(k, l)} \leq 0, \\
l=1,2, \ldots, m, \quad k=1,2, \ldots, m, \quad k \neq l .
\end{gathered}
$$

$\left(\mathbf{X}_{0}, \widehat{\mathbf{Y}}_{\mathbf{0}}\right)$ is the feasible solution of model (11) and $\left(\begin{array}{c}\mathbf{X}_{0} \\ -\widehat{\mathbf{Y}}_{0}\end{array}\right) \leq$ $\left(\begin{array}{c}\mathrm{X}_{0} \\ -\mathrm{Y}_{0}\end{array}\right)$. The conclusion is contrary to the hypothesis before.

Then the hypothesis is not set up, and if $\left(\mathbf{X}_{\mathbf{0}}, \mathbf{Y}_{\mathbf{0}}\right)$ is the Pareto solution of model (11), the $\mathbf{D M U}_{j_{0}}\left(j_{0} \in\{1,2, \ldots n\}\right)$ is DEA efficient.

\section{Conflict of Interests}

The authors declare that there is no conflict of interests regarding the publication of this paper.

\section{References}

[1] A. Charnes, W. W. Cooper, and E. Rhodes, "Measuring the efficiency of decision making units," European Journal of Operational Research, vol. 2, no. 6, pp. 429-444, 1978.

[2] A. Charnes, W. W. Cooper, B. Golany, L. Seiford, and J. Stutz, "Foundations of data envelopment analysis for ParetoKoopmans efficient empirical production functions," Journal of Econometrics, vol. 30, no. 1-2, pp. 91-107, 1985.

[3] A. Charnes, W. Cooper, and Q. L. Wei, "A semi-infinite multicriteria programming approach to Data Envelopment Analysis," Management Science, vol. 9, pp. 1078-1092, 1984.

[4] J. K. Sengupta, "A fuzzy systems approach in data envelopment analysis," Computers \& Mathematics with Applications, vol. 24, no. 8-9, pp. 259-266, 1992.

[5] T. R. Sexton and H. F. Lewis, "Two-stage DEA: an application to major league baseball," Journal of Productivity Analysis, vol. 19, no. 2-3, pp. 227-249, 2003. 
[6] R. Färe and S. Grosskopf, "Network DEA," Socio-Economic Planning Sciences, vol. 34, no. 1, pp. 35-49, 2000.

[7] P.-C. Chen, C.-C. Chang, M.-M. Yu, and S.-H. Hsu, "Performance measurement for incineration plants using multiactivity network data envelopment analysis: the case of Taiwan," Journal of Environmental Management, vol. 93, no. 1, pp. 95-103, 2012.

[8] Y. Chen, L. Liang, F. Yang, and J. Zhu, "Evaluation of information technology investment: a data envelopment analysis approach," Computers and Operations Research, vol. 33, no. 5, pp. 1368-1379, 2006.

[9] Y. Chen, L. Liang, and J. Zhu, "Equivalence in two-stage DEA approaches," European Journal of Operational Research, vol. 193, no. 2, pp. 600-604, 2009.

[10] H. Fukuyama and W. L. Weber, "A slacks-based inefficiency measure for a two-stage system with bad outputs," Omega, vol. 38, no. 5, pp. 398-409, 2010.

[11] C. Kao and S.-N. Hwang, "Efficiency decomposition in twostage data envelopment analysis: an application to non-life insurance companies in Taiwan," European Journal of Operational Research, vol. 185, no. 1, pp. 418-429, 2008.

[12] C. Kao, "Efficiency decomposition for general multi-stage systems in data envelopment analysis," European Journal of Operational Research, vol. 232, no. 1, pp. 117-124, 2014.

[13] L. Liang, Z.-Q. Li, W. D. Cook, and J. Zhu, "Data envelopment analysis efficiency in two-stage networks with feedback," IIE Transactions, vol. 43, no. 5, pp. 309-322, 2011.

[14] Q. L. Wei and T.-S. Chang, "Optimal system design seriesnetwork DEA models," Journal of the Operational Research Society, vol. 62, no. 6, pp. 1109-1119, 2011.

[15] G. Bi, J. Ding, Y. Luo, and L. Liang, "Resource allocation and target setting for parallel production system based on DEA," Applied Mathematical Modelling, vol. 35, no. 9, pp. 4270-4280, 2011.

[16] R. Y. Duan, P. Tian, and W. P. Zhang, "DEA models with independent subsystems and their application," Journal of Industrial Engineering/Engineering Management, vol. 20, no. 1, pp. 27-31, 2006.

[17] C. Kao, "Efficiency measurement for parallel production systems," European Journal of Operational Research, vol. 196, no. 3, pp. 1107-1112, 2009.

[18] C. Kao and P.-H. Lin, "Qualitative factors in data envelopment analysis: a fuzzy number approach," European Journal of Operational Research, vol. 211, no. 3, pp. 586-593, 2011.

[19] N. Rogge and S. de Jaeger, "Evaluating the efficiency of municipalities in collecting and processing municipal solid waste: a shared input DEA-model," Waste Management, vol. 32, no. 10, pp. 1968-1978, 2012.

[20] Y. S. Yang, B. J. Ma, and M. Koike, "Efficiency-measuring DEA model for production syst em with k independent subsystems," Journal of the Operations Research Society of Japan, vol. 43, no. 3, pp. 343-354, 2000.

[21] H. Amatatsu and T. Ueda, "Input-output tables and network DEA: efficiencies of the 47 prefectures of Japan," in Proceedings of the DEA Symposium, Report, Osaka University, 2009.

[22] C.-H. Wang, Y.-H. Lu, and C.-W. Huang, "R\&D, productivity, and market value: an empirical study from high-technology firms," Omega, vol. 41, no. 1, pp. 143-155, 2013.

[23] Y. Zhao, K. Triantis, P. Murray-Tuite, and P. Edara, "Performance measurement of a transportation network with a downtown space reservation system: a network-DEA approach,"
Transportation Research Part E: Logistics and Transportation Review, vol. 47, no. 6, pp. 1140-1159, 2011.

[24] Y.-H. Chiang, E. W.-L. Cheng, and B.-S. Tang, "Examining repercussions of consumptions and inputs placed on the construction sector by use of I-O tables and DEA," Building and Environment, vol. 41, no. 1, pp. 1-11, 2006.

[25] B. Jiang, S. Liu, and Q. Wang, "Integration of DEA with input-output tables for national economy efficiency analysis," International Journal of Services, vol. 1, pp. 88-97, 2006. 


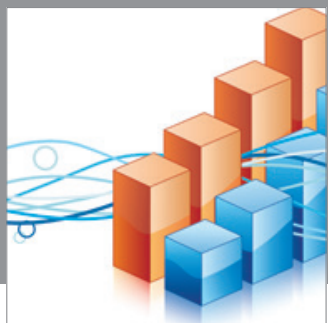

Advances in

Operations Research

mansans

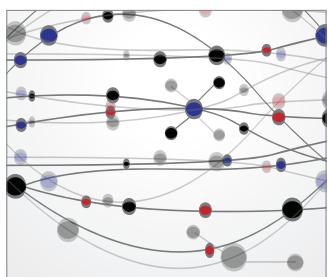

The Scientific World Journal
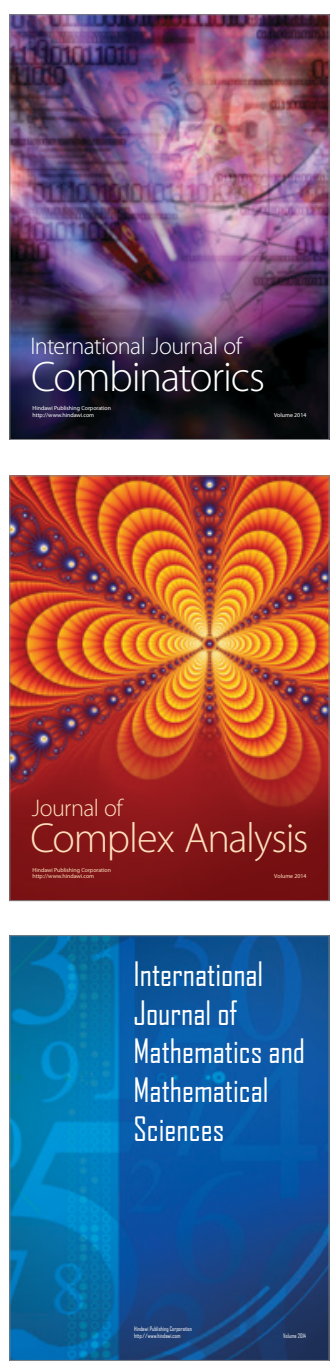
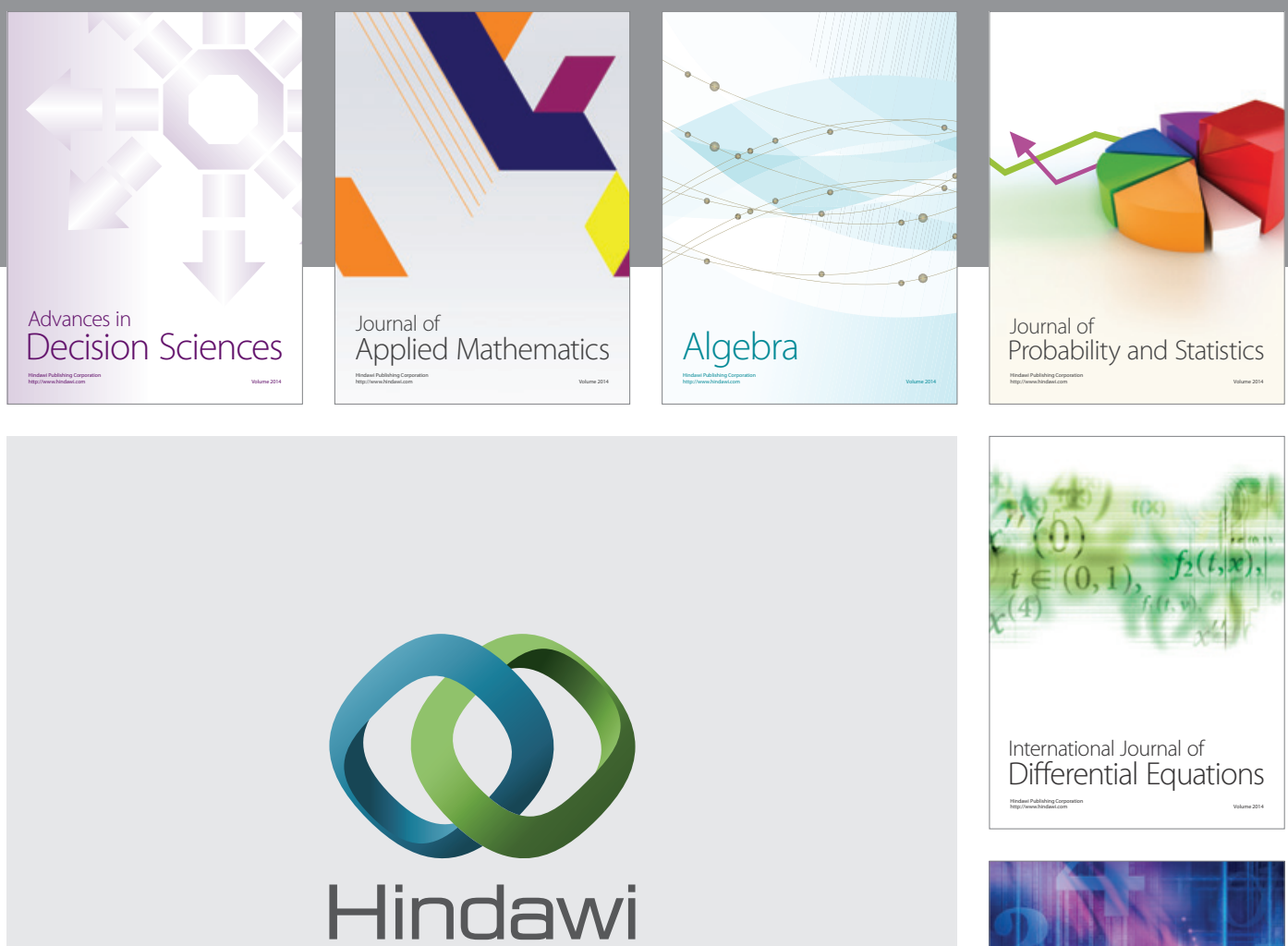

Submit your manuscripts at http://www.hindawi.com
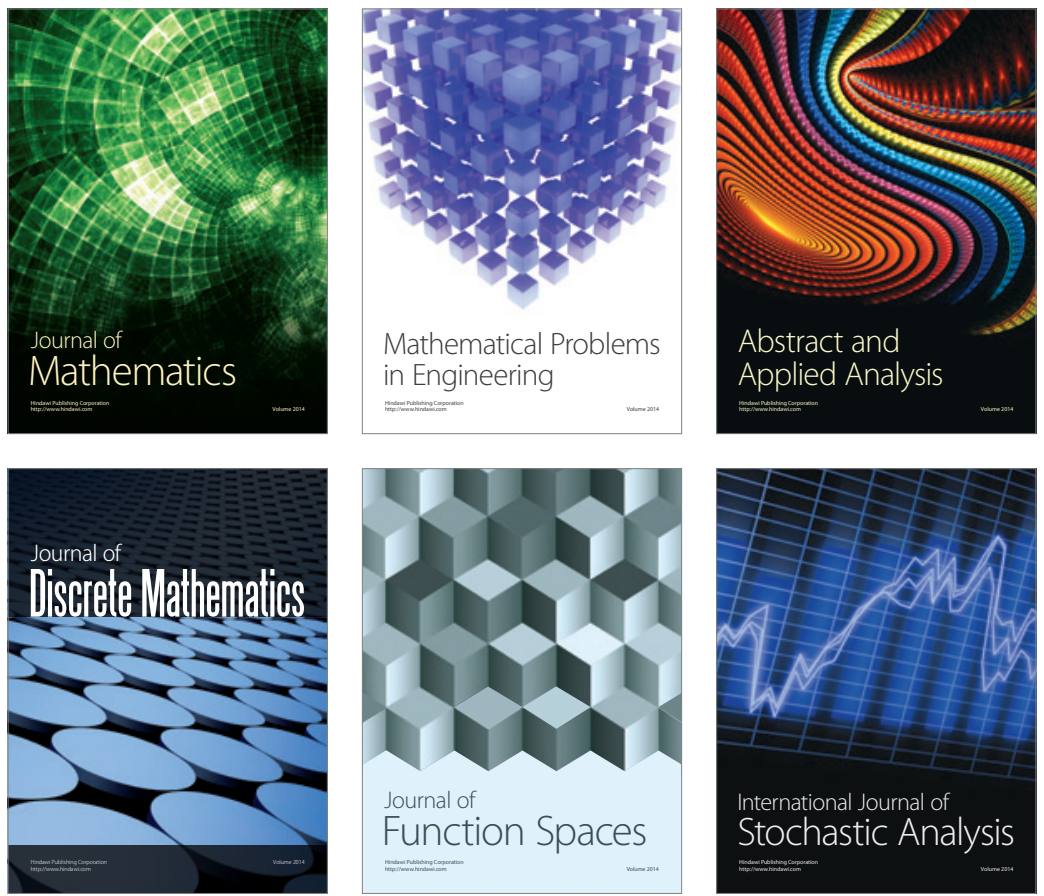

Journal of

Function Spaces

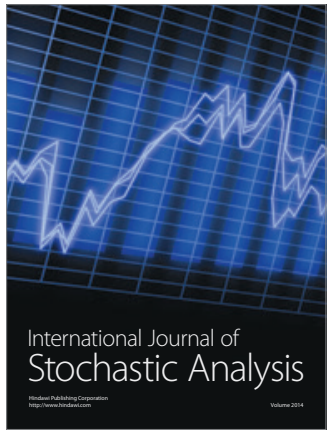

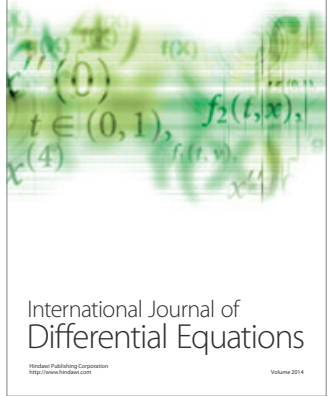
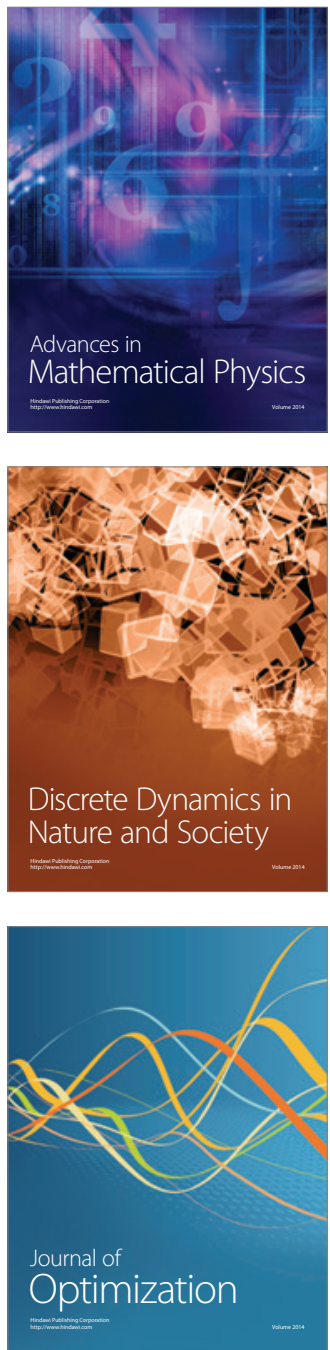